\title{
Avaliação da Capacidade Funcional de Idosos Institucionalizados em um Município de Pequeno Porte do Noroeste do Estado do Rio Grande do Sul
}

\author{
Magliani Reis Fiorin Martel ${ }^{1}$, Gabrieli Della Flora², Ezequiel Vitório Lini ${ }^{3}$
}

\begin{abstract}
RESUMO
Com o aumento da longevidade, é possível observar uma maior parcela de idosos que, muitas vezes, apresentam déficits na capacidade física, mental e cognitiva. Em decorrência deste fenômeno, surgiram as Instituições de Longa Permanência de Idosos (Ilpis), designadas ao cuidado dos idosos, tanto para aqueles que necessitam de uma assistência maior quanto para os que apresentam uma boa independência funcional. Objetivo: Avaliar o grau de capacidade funcional de idosos institucionalizados, identificando suas principais limitações no domínio cognitivo e motor. Métodos: Estudo transversal, quantitativo, descritivo e analítico com 12 idosos residentes da Ilpi em um município do Noroeste do Estado do Rio Grande do Sul. Foram utilizados como instrumentos norteadores um questionário sociodemográfico e a medida de independência funcional (MIF). Resultados: Os idosos institucionalizados apresentaram, na classificação na MIF, independência funcional completa com escore de $116,42 \pm 14,0$, porém o item de domínio cognitivo correspondeu à cognição social, apresentando escore de 16,92 $\pm 3,73$. Conclusão: Os idosos institucionalizados mostraram resultado final de independência completa, porém os escores da cognição social, mais especificamente a resolução de problemas, teve pior desempenho.
\end{abstract}

Palavras-chave: Envelhecimento. Idoso. Atividades cotidianas. Instituição de longa permanência para idosos.

\section{EVALUATION OF THE FUNCTIONAL CAPACITY OF INSTITUTIONALIZED ELDERLY IN A MUNICIPALITY OF SMALL PORTE OF THE NORTHWEST OF THE RIO GRANDE DO SUL STATE}

\section{ABSTRACT}

With increasing longevity, it is possible to observe that a larger proportion of elderly people often present deficits in physical, mental and cognitive capacity. As a result of this phenomenon, Long Term Care Institutions for the Elderly (IIpis) were created, designed to care for the elderly, for those who need greater care and those who have a good functional independence. Objective: To evaluate the functional capacity degree of institutionalized elderly, identifying their main limitations in the cognitive and motor domain. Methods: A cross sectional, quantitative, descriptive and analytical study was performed with 12 elderly Ilpi residents in a city in the Northwest of Rio Grande do Sul State. A sociodemographic questionnaire and the measure of functional independence were used as guiding instruments. Results: The institutionalized elderly presented complete functional independence with a total functional independence score of $116.42 \pm 14.0$, but the item of cognitive domain corresponded to social cognition, presented a score of $16.92 \pm 3.73$. Conclusion: The institutionalized elderly presented complete independence as final result, but the social cognition scores, more specifically problem solving, performed worse. Keywords: Aging. Elderly. Everyday activities. Long stay institution for the elderly.

Recebido em: $17 / 4 / 2017$

Revisões requeridas em: 8/5/2018

Aceito em: 9/10/2018

\footnotetext{
${ }^{1}$ Fisioterapeuta. Mestre em Envelhecimento Humano. Especialista em Fisioterapia Neurofuncional e Preceptoria no Sus. maglifiorin@yahoo.com.br

2 Fisioterapeuta. gabydellaflora@hotmail.com

${ }^{3}$ Mestre em Envelhecimento Humano. ezequielfisio@yahoo.com.br
} 


\section{INTRODUÇÃO}

A longevidade é um fenômeno que encontra-se em constante crescimento nos últimos anos, resultando em modificações na estrutura demográfica. Em 2025, estima- se que o Brasil será o sexto país do mundo com maior número de idosos: aproximadamente 32 milhões de pessoas. Este aumento excessivo gera uma preocupação com a capacidade funcional dos idosos, pois sabe-se que estes possuem uma maior probabilidade de doenças crônicas, as quais podem determinar diferentes formas de incapacidades funcionais (VERAS, 2012).

As complicações que acompanham as doenças crônicas em idosos repercutem negativa e diretamente na qualidade de vida dos idosos, que exigem ações de monitoramento, intervenção e manutenção ou melhora do quadro clínico e funcional (VERAS, 2012).

No envelhecimento há uma perda progressiva da capacidade funcional em decorrência das modificações morfológicas, fisiológicas, psicológicas e bioquímicas. Alguns fatores, comumente presentes nos idosos, contribuem para a instalação de incapacidade funcional, tais como déficits motores e cognitivos, fraqueza muscular e a presença de doenças crônicas não transmissíveis (SMANIOTO; HADDAD, 2011).

A capacidade funcional é definida como a aptidão de realizar as atividades instrumentais de vida diária (AIVD) e atividades básicas de vida diária (ABVD), o que é de extrema importância para ter uma vida autônoma e independente (OLIVEIRA; MENEZES, 2011).

A avaliação da capacidade funcional geriátrica obtém informações do perfil funcional dos idosos, que incluem a análise do desempenho nos cuidados pessoais, de mobilidade e também de interação social (WIBELINGER, 2015). Estes resultados irão auxiliar na estratégia de promoção de saúde, retardando ou prevenindo as incapacidades. Com o aumento da longevidade e o envelhecimento da população, é possível observar uma maior parcela de idosos com déficits na capacidade física, mental e cognitiva (CAMARANO; KANSO, 2010). Essas reduções das habilidades funcionais e cognitivas exigem a presença de cuidados especializados contínuos, que, na maioria das famílias, se tornam inacessíveis financeiramente, e é justamente diante dessas necessidades de cuidados continuados e especializados que surgem as instituições asilares que conhecemos hoje, renomeadas para Instituições de Longa Permanência de Idosos (Ilpis), designadas ao cuidado do idoso (CAMARANO; KANSO, 2010). A composição familiar, dita tradicional, não condiz mais como um único modelo contemporâneo, pois sofreu modificações pela diminuição da taxa de fecundidade, o que tornou as famílias menores. As mulheres estão mais inseridas no mercado de trabalho, e mães solteiras e mulheres e homens vivendo sozinhos reduziu a disponibilidade de cuidadores de idosos, levando a um aumento da procura por Ilpis (KÜCHEMANN, 2012).

Camarano e Kanso (2010) afirmam que coletar dados sobre o grau de capacidade funcional dos residentes em Ilpis é de extrema importância para ocorrer intervenções adequadas, atendendo às necessidades dos idosos. Para a atuação no campo da gerontologia é indispensável a avaliação da capacidade funcional do idoso para haver intervenção funcional. Esta avaliação contribui para que o fisioterapeuta possa ajudar a pessoa idosa, inserindo-a nas atividades de lazer ou desenvolvendo sua capacidade ante as adaptações. Uma boa avaliação fisioterapêutica funcional demonstra o efeito da incapacidade sobre o idoso, que pode ser levemente incômoda até totalmente incapacitante (WIBELINGER, 2015).

O presente estudo teve como objetivo avaliar o grau de capacidade funcional de idosos institucionalizados, identificando suas principais limitações nos domínios cognitivo e motor.

\section{MÉTODOS}

Estudo transversal, quantitativo, descritivo e analítico. A pesquisa foi aprovada pelo Comitê de Ética em Pesquisa com Seres Humanos da Universidade Regional do Noroeste do Estado do Rio Grande do Sul (Unijuí), sob o protocolo no 1.488 .329 , atendendo às diretrizes da Resolução no 466/2012 do Conselho Nacional de Saúde. Todos os participantes receberam informações detalhadas sobre o protocolo de pesquisa e assinaram o Termo de Consentimento Livre e Esclarecido.

Os critérios de inclusão foram idosos de ambos os sexos, com 60 anos ou mais, residentes na Ilpi e que consentiram a pesquisa. Os critérios de exclusão foram: idosos incapazes de se comunicar, os com déficit cognitivo grave, acamados e aqueles que se recusaram a participar da pesquisa.

A instituição onde a pesquisa foi realizada foi a Ilpi Serviço de Amparo e Bem-Estar da Velhice (Sabeve), localizada no município de ljuí no Noroeste do Estado do Rio Grande do Sul, Linha Oeste, BR 522 e km 2. Esta llpi possui uma população de 30 idosos de ambos os sexos. 
Inicialmente foram coletados os dados para caracterização do perfil sociodemográfico, contendo a faixa etária, sexo, cidade, profissão, estado civil, escolaridade, medicações utilizadas e tempo de permanência na instituição.

Para a identificação da capacidade funcional dos idosos aplicou-se o instrumento de Medida de Independência Funcional (MIF), por meio de uma entrevista com perguntas que identificam o nível da capacidade funcional dos idosos. Eles foram convidados a responder o questionário individualmente, sendo coletado pelo próprio fisioterapeuta.

Para Riberto et al. (2001), a MIF tem como objetivo principal quantificar a necessidade de ajuda de terceiros que uma pessoa necessita para realizar suas tarefas motoras e cognitivas. Desta forma, verifica-se o desempenho do idoso em 18 tarefas, divididas em seis dimensões: autocuidado com um total de 42 pontos, subdivididos em alimentação, cuidados com a aparência exterior, banho, habilidade de vestir superior e inferior do corpo e utilização do toalete; o controle de esfíncteres, com total de 14 pontos subdivididos em bexiga e intestino; transferências, com total de 21 pontos subdivididos em leito/cadeira, cadeira de rodas, banho, WC; locomoção, com um total de 14 pontos subdivididos em marcha e escadas; comunicação, com um total de 14 pontos subdivididos em compreensão e expressão; cognição social, com um total de 21 pontos subdivididos em interação social, resolução de problemas e memória.

O nível de independência funcional na MIF é classificado da seguinte forma: Independência completa (7), Independência modificada (6), Supervisão, estímulo ou preparo (5), Assistência mínima (4), Assistência moderada (3), Assistência máxima (2) e Dependência total (1). O escore da escala da MIF varia de 18 a 126 pontos. A pontuação final é classificada em dependência completa (18 pontos), dependência modificada (assistência de até $50 \%$ da tarefa), entre 19-60 pontos, dependência modificada (assistência em até $25 \%$ da tarefa), entre $61-103$ pontos e independência completa, entre 104-126 pontos (RIBERTO et al., 2001).

Após a realização de todas as avaliações, as informações foram tabuladas e digitadas em banco de dados para análise estatística, foi utilizado o programa Statistical Package for Social Sciences (versão 22.0, SPSS, Chicago, Illinois). Os dados foram apresentados em valores absolutos e relativos, média \pm desvio padrão. Para avaliar a normalidade das variáveis foi aplicado o teste Shapiro-Wilk.

\section{RESULTADOS}

A Tabela 1 apresenta as características sociodemográficas da população de idosos institucionalizados, que são 12 indivíduos, seis $(50 \%)$ do sexo feminino e seis (50\%) do sexo masculino, com idade média de 77,42 $\pm 9,00$ anos. Quanto à escolaridade, nenhum dos idosos cursou o Ensino Superior e 58,3\% não concluíram o Ensino Fundamental, sugerindo baixo grau de escolaridade. No quesito situação conjugal, 58,3\% eram viúvos (as). O tempo de institucionalização mostrou-se com distribuição não normal, com média de $12,00 \pm 50,54$, com um tempo mínimo de um mês de residência na instituição e máximo de 156 meses.

Tabela 1 - Características gerais dos idosos institucionalizados ( $n=12$ ), ljuí - RS, 2016

\begin{tabular}{ll}
\hline Variável & $\mathbf{n}(\%)$ \\
\hline Sexo feminino & $6(50)$ \\
Sexo masculino & $6(50)$ \\
$\begin{array}{l}\text { ldade Média } \\
\text { Escolaridade }\end{array}$ & $77,4( \pm 9,0)$ \\
Analfabeto & \\
Fundamental incompleto & $1(8,3)$ \\
Médio completo & $7(58,3)$ \\
Não sabe/não respondeu & $2(16,7)$ \\
Tempo de instituição & $2(16,7)$ \\
Situação conjugal & $12,0( \pm 50,54)$ \\
Viúvo(a) & $7(58,3)$ \\
Solteiro(a) & $3(25,1)$ \\
Divorciado(a) & $1(8,3)$ \\
Casado(a) & $1(8,3)$ \\
\hline \multicolumn{2}{c}{$\quad$ Fonte: Dados da pesquisa. }
\end{tabular}

Nota: Valores expressam frequência absoluta, média ou mediana \pm desvio padrão.

Os valores relacionados à capacidade funcional estão elucidados na Tabela 2. Destaca-se, como resultado geral da escala MIF, correspondendo aos domínios motor e cognitivo o valor de $116,42( \pm 14,00)$, o que permite classificar os idosos institucionalizados em independência completa. Nas dimensões específicas foram encontradas as seguintes médias: no quesito cuidados pessoais 40,33 pontos, controle esfincteriano 13,25, transferências 20,17 , locomoção 12,42, comunicação 13,33 e cognição social 16,92. 
Tabela 2 - Teste de medida de independência funcional de idosos institucionalizados (n=12), ljuí-RS, 2016

\begin{tabular}{ll}
\hline Dimensões específicas & Média \pm DP \\
\hline Cuidados pessoais & $40,33 \pm 5,77$ \\
Controle esfincteriano & $13,25 \pm 2,01$ \\
Transferências & $20,17 \pm 2,88$ \\
Locomoção & $12,42 \pm 2,15$ \\
Comunicação & $13,33 \pm 1,07$ \\
Cognição social & $16,92 \pm 3,73$ \\
MIF total & $116,42( \pm 14,0)$ \\
\hline
\end{tabular}

Fonte: Dados da pesquisa.

Os escores relacionados à cognição social mostraram-se com pior desempenho, e, portanto, optou-se por demonstrá-los separadamente (interação social, resolução de problemas e memória) para a identificação das tarefas de maior comprometimento. Assim, a média da categoria interação social foi de $6,92( \pm 0,29)$, resolução de problemas com média de $4,42( \pm 2,43)$ e memória com média de $5,58( \pm 1,44)$.

\section{DISCUSSÃO}

Neste estudo evidenciou-se que os idosos avaliados preservam a capacidade funcional, sendo classificada, segundo a escala MIF, como independência completa, porém demonstrando melhores resultados no domínio motor e maior comprometimento na cognição social.

A amostragem referente ao gênero no estudo atual apresentou resultados idênticos. Este achado difere da grande parte dos estudos, que referem o predomínio da população feminina nas Ilpis (TOMICKI et al., 2017). Rodrigues et al. (2015) também mostrou um porcentual mais amplo de mulheres, em razão de que estas apresentam uma maior expectativa de vida.

A idade média na população deste estudo permaneceu em $77,42 \pm 9,00$, quando a mínima foi de 60 anos e a máxima de 92 anos. Dados semelhantes foram encontrados na pesquisa de Lisboa e Chianca (2012), em que a idade média encontrada foi de 77 $\pm 8,5$ anos e a maior parcela estava com 80 anos ou mais.

Com relação à escolaridade dos idosos pesquisados, observou-se que 58,3\% não concluíram o Ensino Fundamental, 16,7\% concluíram o Ensino Médio, $8,3 \%$ eram analfabetos e $16,7 \%$ não souberam responder. A baixa escolaridade foi semelhante à identificada por Ferreira et al. (2011) entre idosos residentes de uma Ilpi em Belém (PA), onde 74,5\% possuíam nível fundamental incompleto, 2,1\% Ensino Médio completo e $8,5 \%$ eram analfabetos. De acordo com Alves-
-Silva, Scorsolini-Comin e Santos (2013), o baixo nível de escolaridade entre os idosos possui relação direta com o pouco reconhecimento que o aprendizado escolar apresentava na infância dos mesmos, pois esta não era a principal prioridade da família, o que vai ao encontro dos dados da presente pesquisa.

A situação conjugal encontrada nos idosos da Ilpi estudada foi constituída em uma maior parcela de viúvos $58,3 \%$, seguindo de solteiros $25,1 \%$, divorciados $8,3 \%$ e casados $8,3 \%$. Araújo et al. (2014) avaliaram dez idosos de uma Ilpi localizada no município de Parnaíba (PI), encontrando porcentual semelhante, em que $50 \%$ dos idosos eram separados ou viúvos, $10 \%$ casados e $40 \%$ solteiros. Para Araújo, Neto e Bós (2016), uma das causas do processo de institucionalização acontece em decorrência da viuvez. Muitos destes idosos não possuem filhos ou parentes para recorrer e outros referem receio em sobrecarregar familiares após a perda de um cônjuge.

No presente estudo, o tempo mínimo de institucionalização encontrado foi de um mês e o tempo máximo de 156 meses, resultando em média de 12,00 $\pm 50,54$ meses. Isso se deve ao fato de que a qualquer momento um idoso pode ser alocado na instituição. Segundo Lisboa e Chianca (2012), o tempo de institucionalização dos idosos foi, em média, de 70 meses, variando de 1 a 328 meses. A semelhança encontrada entre as pesquisas se direciona ao fato de que as Ilpis se tornam, muitas vezes, a única alternativa viável de residência para o longevo, os quais geralmente enveIhecem e permanecem no local até seus últimos dias de vida.

A MIF foi utilizada como instrumento de avaliação na pesquisa de Ferreira et al. (2011) para identificar a capacidade funcional de idosos institucionalizados. Os resultados encontrados demonstraram que esta população específica estava classificada em independência completa/modificada, com escore final de $109,7 \pm 9,3$. Este achado vai de encontro aos obtidos no estudo, no qual, apesar da idade avançada de alguns idosos, prevaleceu a independência completa com um escore de $116,42 \pm 14,0$. Contribuindo com os resultados supracitados, dados da pesquisa de Guedes e Silveira (2004), realizada com 109 idosos institucionalizados, identificaram a prevalência de independência em 59,63\% dos idosos da população estudada.

Trindade et al. (2013) tiveram como objetivo analisar o declínio da cognição e o seu impacto nas habilidades funcionais em idosos institucionalizados e não institucionalizados. Os achados foram similares aos do estudo atual. Os resultados encontrados permitem concluir que a baixa escolaridade e a institucio- 
nalização contribuem, de forma crucial, para a degradação cognitiva e futuramente ao comprometimento das habilidades funcionais.

Ao utilizar a MIF, Macêdo et al. (2012) observaram que o domínio cognitivo, nas dimensões da cognição social (memória, resolução de problemas e interação social), apresentou um escore 16,0 $\pm 4,8$; a idade dos idosos era superior a 80 anos. Também descrevem que idosos com declínio cognitivo podem ter maior tendência para desenvolver uma dependência funcional futura e, consequentemente, a perda de autonomia. $\mathrm{O}$ artigo vai ao encontro dos recentes achados da pesquisa, quando o escore final deste subitem foi de $16,92 \pm 3,73$, apresentando pior desempenho no que se refere à resolução de problemas.

$\mathrm{Na} \mathrm{MIF}$, o domínio cognitivo envolve cinco atividades funcionais distintas, sendo este um dos maiores diferenciais desta escala de avaliação, pois, geralmente, este quesito é avaliado em testes neuropsicológicos. No contexto de cada atividade, a interação social envolve a capacidade da pessoa em lidar com suas próprias necessidades em conjunto com as necessidades dos outros. A resolução de problemas abrange a pessoa resolver os seus problemas de vida diária, sendo capaz, assim, de tornar suas devidas decisões. A capacidade de guardar e recuperar informações, reconhecimento e lembranças de rotinas esta correlacionado ao tópico memória (RIBERTO et al., 2001, 2004).

Durante a avaliação, evidenciou-se que os idosos da instituição apresentam uma autonomia limitada para a resolução dos seus problemas diários; alguns relatos foram de perda de autonomia para administrar seus próprios bens de forma independente.

A institucionalização e a redução da cognição podem ser hipóteses determinantes para a perda da capacidade de resolução de problemas. Sendo assim, é possível afirmar, contribuindo com outros autores, que alterações cognitivas podem futuramente ser fatores agravantes para o desenvolvimento de maior dependência para suas atividades de vida diárias.

As instituições podem determinar incapacidades para gerir a própria vida. Segundo Alves-Silva, Scorsolini-Comin e Santos (2013), as Ilpis apresentam regras cotidianas preestabelecidas. Em razão da vida padronizada e da expectativa de vida diminuída, os idosos perdem o direito de se expressar, tendo, assim, a sua vida limitada social e afetivamente, ficando suscetíveis a desenvolver a dependência funcional. Nas Ilpis raramente encontram-se atividades para idosos autônomos e independentes, uma vez que para essa efetivação acontecer seria necessária a integração de uma equipe multiprofissional dentro da instituição com o objetivo de manter o idoso ativo para conduzir sua vida de acordo com o cotidiano em que está inserido.

Os resultados encontrados, contudo, reafirmam que a capacidade funcional é considerada um fator fundamental no processo de envelhecimento saudável, podendo retardar os agravos e as incapacidades verificadas neste estudo. Cabe ressaltar que esta pesquisa apresentou algumas limitações que não permitem a generalização desses resultados; também por ser um estudo local, o qual identifica a real situação exclusiva de um município em questão, além de ter sido realizado com um número reduzido de idosos.

\section{CONCLUSÃO}

O término desta pesquisa possibilitou evidenciar que a maioria dos idosos institucionalizados apresentou como resultado final independência completa segundo a escala da MIF, porém os escores relacionados com a cognição social, mais especificamente a resolução de problemas, demonstraram pior desempenho.

Avaliar e identificar as características da capacidade funcional nessa população pesquisada, entretanto, é de grande relevância para manter a integridade física e mental. Somente a partir de uma clara identificação das principais incapacidades funcionais presentes nos idosos, bem como a compreensão dos fatores desencadeantes, é que será possível prevenir ou retardar a sua instalação. $O$ instrumento MIF pode ser utilizado como condutor de tomadas de decisão para condutas e intervenções adequadas, buscando aperfeiçoar as funções remanescentes e, se possível, recuperar as incapacidades perdidas.

\section{REFERÊNCIAS}

ALVES-SILVA, J. D.; SCORSOLINI-COMIN, F.; SANTOS, M. A. $D$. Idosos em instituições de longa permanência: desenvolvimento, condições de vida e saúde. Psicologia: Reflexão $e$ Crítica, Porto Alegre, v. 26, n. 4, p. 820-830, 2013.

ARAÚJO, A. M.; NETO, S. T. B.; BÓS, A. J. G. Diferenças no perfil de pessoas idosas institucionalizadas, em lista de espera e que não desejam institucionalização. Revista Brasileira de Geriatria e Gerontologia, Rio de Janeiro, v. 19, n. 1, p. 105-118, 2016.

ARAÚJO, R. S. S. et al. Institutionalized Elderly: Clinical and Functional Profile. Revista de Enfermagem da Ufpi, Piauí, v. 3, n. 2, p. 69-77, 2014.

CAMARANO, A. A.; KANSO, S. As instituições de longa permanência para idosos no Brasil. Revista Brasileira de Estudos de População, Rio de Janeiro, v. 27, n. 1, p. 232-235, 2010. 
FERREIRA, T. C. D. R. et al. Análise da capacidade funcional de idosos institucionalizados. Revista Brasileira de Ciências do envelhecimento Humano, Passo Fundo, v. 8, n. 1, p. 9-20, 2011.

GUEDES, J. M.; SILVEIRA, R. C. R. Análise da capacidade funcional da população geriátrica institucionalizada na cidade de Passo Fundo - RS. Revista Brasileira de Ciências do envelhecimento Humano, Passo Fundo, v. 10, n. 21, p. 1021, 2004.

KÜCHEMANN, B. A. Envelhecimento populacional, cuidado e cidadania: velhos dilemas e novos desafios. Sociedade $e$ Estado, Brasília, DF, v. 27, n. 1, p. 165-180, 2012.

LISBOA, C. R.; CHIANCA, T. C. M. Perfil epidemiológico, clínico e de independência funcional de uma população idosa institucionalizada. Revista Brasileira de Enfermagem, Brasília, v. 65, n. 3, p. 482-487, 2012.

MACÊDO, A. M. L. et al. Avaliação funcional de idosos com déficit cognitivo. Acta Paulista de Enfermagem, São Paulo, v. 25, n. 3, p. 358-363, 2012.

OLIVEIRA, L. P. B. A. D.; MENEZES, R. M. P. D. Representações de fragilidade para idosos no contexto da estratégia saúde da família. Texto \& Contexto Enfermagem, Florianópolis, v. 20, n. 2, p. 301-309, 2011.

RIBERTO, M. et al. Reprodutibilidade da versão brasileira da Medida de Independência Funcional. Acta fisiátrica, São Paulo, v. 8, n. 1, p. 45-52, 2001.
RIBERTO, M. et al. Validação da versão brasileira da Medida de Independência Funcional. Acta Fisiátrica, São Paulo, v. 11, n. 2, p. 72-76, 2004.

RODRIGUES, W. K. M. et al. Atividade física e incapacidade funcional em idosos da zona rural de um município do Nordeste do Brasil. Revista Brasileira em Promoção da Saúde, Fortaleza, v. 28, n. 1, p. 126-132, 2015.

SMANIOTO, F. N.; HADDAD, M. C. F. L. Índice de Katz aplicado a idosos institucionalizados. Revista Rene, Fortaleza, v. 12, n. 1, p. 18-23, 2011.

TOMICKI, C. et al. Associação entre número de quedas e força muscular de idosos residentes em instituições de longa permanência. Revista Kairós ? Gerontologia, v. 20, n. 2, p. 101-116, 2017.

TRINDADE, A. P. N. T. et al. Repercussão do declínio cognitivo na capacidade funcional em idosos institucionalizados e não institucionalizados. Fisioterapia em Movimento, v. 26, n.2, p. 81-89, 2013.

VERAS, R. P. Um modelo em que todos ganham: mudar e inovar, desafios para o enfrentamento das doenças crônicas entre os idosos. Acta Scientiarum. Human and Social Sciences, Maringá, v. 34, n. 1, p. 3-8, 2012.

WIBELINGER, L. M. Avaliação em fisioterapia geriátrica. In: WIBELINGER, L. M. Fisioterapia em Geriatria. 1. ed. Rio de Janeiro: Revinter, 2015. p. 1-37. 